\title{
Correction to: Logic and Society
}

\section{Correction to}

(C) The Author(s) 2018

Y. Kawana, Logic and Society, https://doi.org/10.1057/978-1-137-52221-4

The book was inadvertently published, before incorporating necessary changes in the proofs. The version supplied here has been corrected and approved by the author.

The updated original online version of this book can be found at https://doi.org/10.1057/978-1-137-52221-4 